\title{
Few-mode multi-element fiber amplifier for mode division multiplexing
}

\author{
S. Jain," Y. Jung, T. C. May-Smith, S. U. Alam, J. K. Sahu, and D. J. Richardson \\ Optoelectronics Research Centre, University of Southampton, Southampton, SO17 1BJ, UK \\ *sj3g11@orc.soton.ac.uk
}

\begin{abstract}
We experimentally demonstrate a few-moded cladding-pumped multi-element fiber amplifier, comprising $4 \mathrm{Er} / \mathrm{Yb}$ co-doped signal fibers and 1 multimode pump-delivery fiber all of which are drawn together in a common polymer coating, providing a total spatial path multiplicity of 12 . An average WDM signal gain of $18.3 \mathrm{~dB}$ and differential modal gain of 1.1 $\mathrm{dB}$ were achieved in the spectral range of $1542-1560 \mathrm{~nm}$.
\end{abstract}

(C)2014 Optical Society of America

OCIS codes: (060.2320) Fiber optics amplifiers and oscillators; (060.0060) Fiber optics and optical communications.

\section{References and links}

1. R. W. Tkach, "Scaling optical communications for next decade and beyond," Bell Labs Technical Journal. 14(4), 3-9 (2010).

2. D. J. Richardson, J. M. Fini, and L. E. Nelson, "Space-division multiplexing in optical fibres," Nat. Photonics 7(5), 354-362 (2013).

3. R. Ryf, S. Randel, A. H. Gnauck, C. Bolle, A. Sierra, S. Mumtaz, M. Esmaeelpour, E. C. Burrows, R. Essiambre, P. J. Winzer, D. W. Peckham, A. H. McCurdy, and R. Lingle, "Mode-division multiplexing over $96 \mathrm{~km}$ of fewmode fiber using coherent $6 \times 6$ MIMO processing," J. Lightwave Technol. 30(4), 521-531 (2012).

4. Y. Jung, Q. Kang, V. A. J. M. Sleiffer, B. Inan, M. Kuschnerov, V. Veljanovski, B. Corbett, R. Winfield, Z. Li, P. S. Teh, A. Dhar, J. K. Sahu, F. Poletti, S.-U. Alam, and D. J. Richardson, "Three mode Er3+ ring-doped fiber amplifier for mode-division multiplexed transmission," Opt. Express 21(8), 10383-10392 (2013).

5. M. Koshiba, K. Saitoh, and Y. Kokubun, "Heterogeneous multi-core fibres: proposal and design principle," IEICE Electron. Express 6(2), 98-103 (2009).

6. J. Sakaguchi, W. Klaus, B. J. Puttnam, J. M. D. Mendinueta, Y. Awaji, N. Wada, Y. Tsuchida, K. Maeda, M. Tadakuma, K. Imamura, R. Sugizaki, T. Kobayashi, Y. Tottori, M. Watanabe, and R. V. Jensen, "19-core MCF transmission system using EDFA with shared core pumping coupled via free-space optics," Opt. Express 22(1), 90-95 (2014)

7. K. S. Abedin, J. M. Fini, T. F. Thierry, B. Zhu, M. F. Yan, L. Bansal, F. V. Dimarcello, E. M. Monberg, and D. J. DiGiovanni, "Seven-core erbium-doped double-clad fiber amplifier pumped simultaneously by side-coupled multimode fiber," Opt. Lett. 39(4), 993-996 (2014).

8. V. Sleiffer, Y. Jung, N. Baddela, J. Surof, M. Kuschnerov, V. Veljanovski, J. Hayes, N. Wheeler, E. Numkam Fokoua, J. Wooler, D. Gray, N. Wong, F. Parmigiani, S. Alam, M. Petrovich, F. Poletti, D. Richardson, and H. de Waardt, "High capacity mode-division multiplexed optical transmission in a novel 37-cell hollow-core photonic bandgap fiber," J. Lightwave Technol. 32(4), 854-863 (2014).

9. S. Jain, V. J. F. Rancano, T. C. May-Smith, P. P. Petropoulous, J. K. Sahu, and D. J. Richardson, "Multi-element fiber technology for space division multiplexing application," Opt. Express 22(4), 3787-3796 (2014).

10. S. Jain, T. C. May-Smith, A. Dhar, A. S. Webb, M. Belal, D. J. Richardson, J. K. Sahu, and D. N. Payne, "Erbium-doped multi-element fiber amplifiers for space-division multiplexing operations," Opt. Lett. 38(4), 582-584 (2013).

11. S. Jain, T. C. May-Smith, and J. K. Sahu, "Er/Yb-doped Cladding-Pumped Multi-Element Fiber Amplifier" in Workshop on Specialty Optical Fiber, 5.4, Sigtuna (2013).

12. S. Jain, T. C. May-Smith, and J. K. Sahu, "Cladding-pumped Er/Yb-doped Multi-Element Fiber Amplifier for C+L band Operations," in Optical Fiber Communications, M2J.3, San Francisco (2014).

13. T. Mizuno, T. Kobayashi, H. Takara, A. Sano, H. Kawakami, T. Nakagawa, Y. Miyamoto, Y. Abe, T. Goh, M. Oguma, T. Sakamoto, Y. Sasaki, I. Ishida, K. Takenaga, S. Matuso, K. Saitoh, and T. Morioka, "12-core x 3mode dense space division multiplexed transmission over $40 \mathrm{~km}$ employing multi-carrier signals with parallel MIMO equalization," in Optical Fiber Communications, Th5B.2, San Francisco (2014).

14. Y. Sasaki, K. Takenaga, S. Matsuo, K. Saitoh, and M. Koshiba, "Trench-assisted low-crosstalk few-mode multicore fiber," in European Conference on Optical Communications, Mo.3.A.5, London, (2013). 
15. C. Xia, R. A. Correa, B. Neng, E. A. Lopez, D. M. Arrioja, A. Schulzgen, M. Richardson, J. Linares, C. Montero, E. Mateo, X. Zhao, and G. Li, "Hole-assisted few-mode multicore fiber for high-density space-division multiplexing," IEEE Photon. Technol. Lett. 24(21), 1914-1917 (2012).

16. R. Horley, S. Norman, and M. N. Zervas, "Progress and development in fibre laser technology," Proc. SPIE 6738, 67380K (2007), doi:10.1117/12.753171.

17. E. L. Lim, Y. Jung, Q. Kang, T. C. May-Smith, N. H. L. Wong, R. Standish, F. Poletti, J. K. Sahu, S. U. Alam, and D. J. Richardson, "First demonstration of cladding pumped few-moded EDFA for mode division multiplexed transmission," in Optical Fiber Communications, M2J.2, San Francisco (2014).

\section{Introduction}

Space division multiplexing (SDM) [1-3] has generated significant interest within the fiber optics community as the next potential breakthrough technology to address the anticipated telecoms 'capacity crunch'. Various approaches such as multi-mode fiber (MMF) [3], ringcore fiber [4], multicore fiber (MCF) [5-7], hollow-core photonic bandgap fiber [8], and multi-element fiber (MEF) [9-12] have been introduced over the past few years to enable the development of high capacity transmission networks using multiple spatial channels. Recently, fiber technologies combining two or more of the above mentioned approaches have been proposed in order to further increase the transmission capacity. Few-mode multicore fiber is one such example whereby each core in a MCF can accommodate multiple modes, and a spatial multiplicity of 36 was recently demonstrated, employing 3-modes per core in a 12-core MCF [13]. Distributed inter-core crosstalk is a primary concern in any SDM systems based on MCF and, whilst trench assisted and hole assisted core designs offer reduced levels of cross-talk and hence increased core densities [14,15], mechanical reliability issues constrain the maximum outer diameter of the fiber and hence the ultimate achievable spatial multiplicity. The development of complex, high performance multiplexers/demultiplexers, as well as accurate splicing is also essential to ensure low levels of crosstalk between neighboring cores. Moreover, until now, SDM amplifiers supporting few-mode MCFs have not yet been demonstrated. MEF, comprising multiple fiber-elements in a common polymer coating appears an attractive alternative to MCFs in many instances due to the ultralow crosstalk levels (less than $-80 \mathrm{~dB}$ in $9.5 \mathrm{~km}$ of passive MEF [9]) between individual cores, simple fan-in, fan-out multiplexing (access to individual cores is obtained simply by stripping-off the polymer coating) and practicality in terms of amplifier construction. We have previously fabricated and characterized passive single-mode MEFs for SDM data transmission [9]. Both core-pumped and cladding-pumped MEF amplifiers have also been demonstrated, with up to 7 fibers in a single assembly [10-12]. In order to increase the capacity for future SDM networks, the number of spatial channels needs to be further increased and the introduction of a few-mode core in a MEF structure can help further increase the capacity by taking the benefit of two spatial multiplexing schemes (MMF and MEF). To realize such an SDM system, a few-mode MEF amplifier is an essential subsystem to be developed.

In this paper, we present the first demonstration of a 3-moded cladding-pumped MEF amplifier comprising 5 fiber elements $(4 \mathrm{Er} / \mathrm{Yb}$ doped signal fiber-elements and 1 central multimode pump-delivery fiber-element) to scale up the spatial multiplicity. Pump couples into the signal fiber-elements via evanescent coupling throughout the length of the fiber if optical contact is maintained [16]. It was verified that the signal fiber-elements maintained optical contact by inspecting the cross-section image at multiple points in $4 \mathrm{~m}$ of fiber length. Each fiber element can amplify three spatial modes $\left(\mathrm{LP}_{01}, \mathrm{LP}_{11 \mathrm{a}}\right.$, and $\left.\mathrm{LP}_{11 \mathrm{~b}}\right)$ yielding an overall multiplicity of 12 ( 3 modes x 4 signal fiber-elements). An average signal gain of $18.3 \mathrm{~dB}$ and differential modal gain in the range of $\sim 1-6 \mathrm{~dB}$ were achieved in the wavelength range $1542-1560 \mathrm{~nm}$ at an input signal power of $-12.5 \mathrm{dBm}$ per channel. The detailed WDM performance for various pump powers and input signal powers has also been investigated. 


\section{Experiment setup of 3M-MEFA}

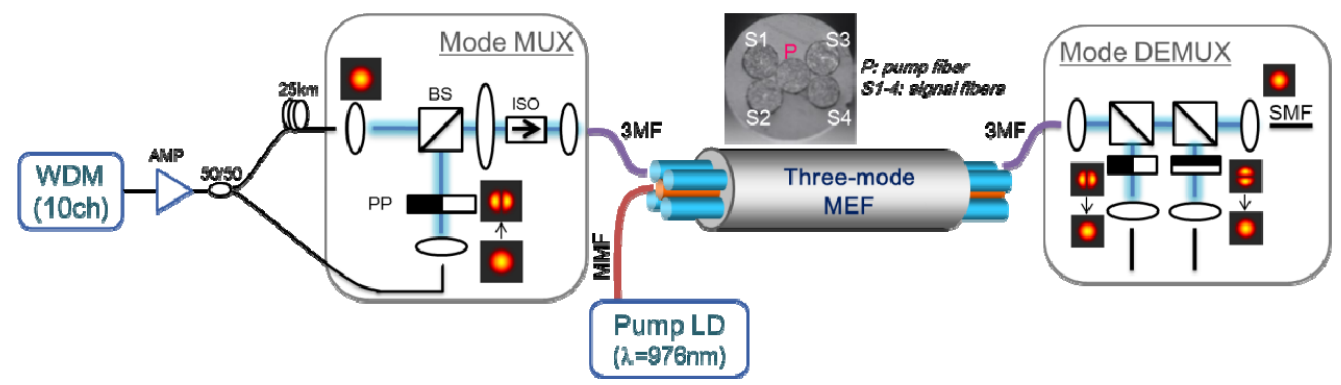

Fig. 1. Schematic diagram of 3-moded cladding pumped multi-element fiber amplifier (3MMEFA) comprising $4 \mathrm{Er} / \mathrm{Yb}$ co-doped signal fibers and 1 multimode pump fiber. (BS: Beam splitter, PP: Phase plate, ISO: isolator, AMP: amplifier, 3MF: 3-moded passive fiber).

Figure 1 shows the schematic of the setup used for the characterization of the 3-moded cladding pumped MEF amplifier (3M-MEFA). Each signal fiber-element has a cladding diameter of $80 \mu \mathrm{m}$ and a core diameter of $\sim 8 \mu \mathrm{m}$ with a step-index refractive index profile (NA 0.17-0.20), which can guide up to three spatial modes $\left(\mathrm{LP}_{01}, \mathrm{LP}_{11 \mathrm{a}}\right.$, and $\left.\mathrm{LP}_{11 \mathrm{~b}}\right)$. The fiber-elements were coated with a common low-index polymer coating $(\mathrm{n} \sim 1.373)$ to obtain a double-clad fiber design and thereby enabling the possibility to use a low cost, high-power, multimode laser diode as a pump laser operating at a wavelength of $976 \mathrm{~nm}$. The cores of the signal fiber-elements were co-doped with $\mathrm{Yb}$ to enhance pump absorption. A cladding pump absorption of $2.2-4.1 \mathrm{~dB} / \mathrm{m}$ was measured at $976 \mathrm{~nm}$. The signal source used in the setup consisted of 10 wavelength multiplexed external cavity lasers spread over the range 1536$1560 \mathrm{~nm}$. The wavelength division multiplexed (WDM) channels were pre-amplified, split into two and fed into the mode multiplexer to allow the gain performance of the individual amplifier elements to be measured. A $25 \mathrm{~km}$ single mode fiber span was inserted along the $\mathrm{LP}_{01}$ path to minimize modal beating due to the high degree of coherence between the seed lasers. A phase-plate based mode multiplexer was used to selectively excite the pure $\mathrm{LP}_{01}$ and $\mathrm{LP}_{11}$ signal modes in a $10 \mathrm{~m}$ long passive 3 -moded fiber (3MF). The passive fiber (a graded index fiber with $16 \mu \mathrm{m}$ core diameter and an NA of 0.14, OFS Denmark) was then spliced directly to one of the Er/Yb-doped elements of the 3M-MEF. The amplified output was fed into the mode demultiplexer to analyze the mode dependent gain quantitatively. The large mode field diameter mismatch between the passive and active fibers resulted in a mode dependent splice loss $\left(\sim 1.1 \mathrm{~dB}\right.$ for the $\mathrm{LP}_{01}$ and $\sim 2.5 \mathrm{~dB}$ for the $\mathrm{LP}_{11}$ mode). The $105 / 125 \mu \mathrm{m}$ pigtail of the $976 \mathrm{~nm}$ multimode pump laser was adiabatically tapered down to $80 \mu \mathrm{m}$ and fusion spliced to the central pump fiber element of the 3M-MEF. The pump light gradually coupled from the pump element into the 4 surrounding $\mathrm{Er} / \mathrm{Yb}$-doped fiber elements through evanescent field coupling and is steadily absorbed by the rare-earth ions within the FMF cores. 
(a) before amplification
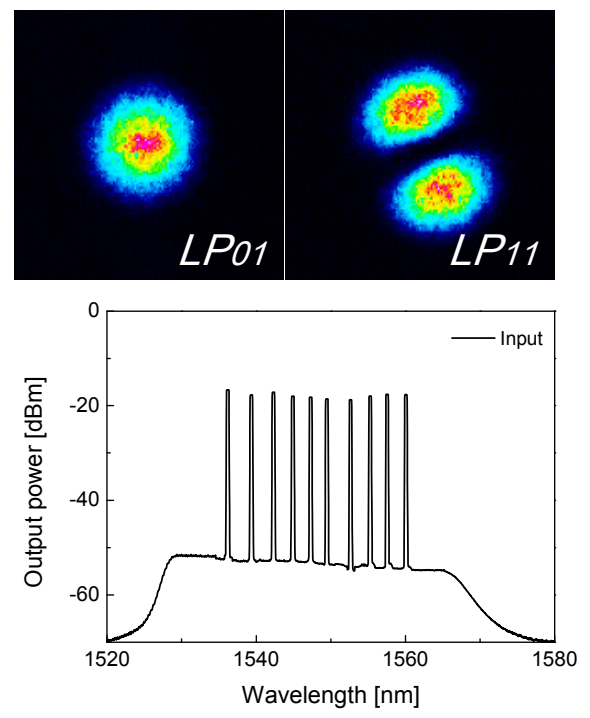

(b) after amplification
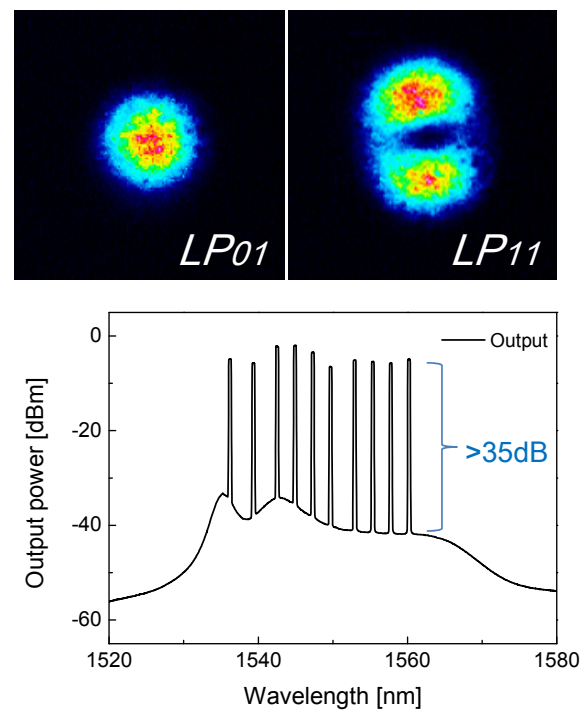

Fig. 2. Measured mode images and optical spectra before (a) and after (b) amplification.

To confirm clean mode amplification of the input signals, mode images were taken using a charge coupled device (CCD) camera before and after amplification at $1550 \mathrm{~nm}$. The top row of Fig. 2 shows that two clean spatial modes $\left(\mathrm{LP}_{01}\right.$ and $\left.\mathrm{LP}_{11}\right)$ were excited in the passive $3 \mathrm{MF}$ and that the mode quality was well preserved during amplification. The bottom row of Fig. 2 shows the optical spectra of the WDM signals $\left(\mathrm{LP}_{01}\right)$ before and after the 3M-MEFA demonstrating more than $35 \mathrm{~dB}$ optical signal to noise ratio (OSNR) after amplification. The length of the amplifier was chosen to be $3.25 \mathrm{~m}$ in order to maximize the signal gain whilst maintaining near full C-band operation. The gain peak of the amplifier was located at 1545 $\mathrm{nm}$.

\section{Gain performance of 3M-MEFA}

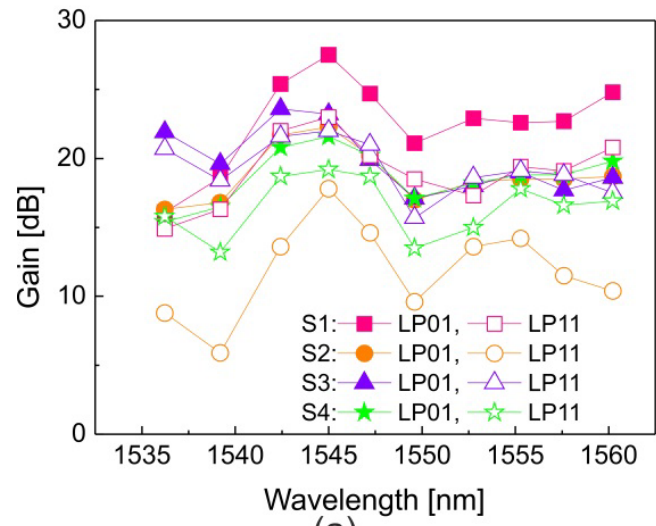

(a)

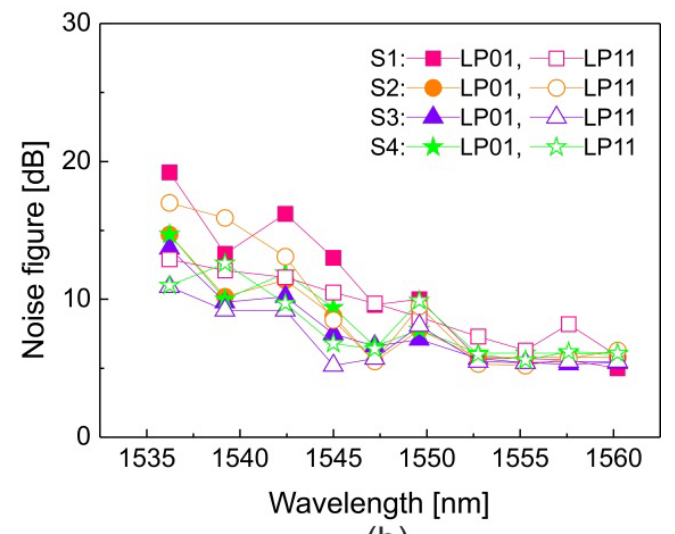

(b)

Fig. 3. (a) Gain and (b) noise figure spectra for 4 different signal fiber elements of the 3MMEFA for an input signal power of $-12.5 \mathrm{dBm} / \mathrm{ch}$ and pump power of $\sim 7.6 \mathrm{~W}$.

Figure 3(a) and 3(b) shows the WDM gain and noise figure (NF) spectra for all 4 different signal fiber elements of the 3M-MEFA, referred as S1 to S4, measured consecutively over all 
active fiber elements. A maximum pump power of $7.6 \mathrm{~W}$ and an input signal power of $-2.5 \mathrm{dBm}$ per mode (total signal input power of $0.5 \mathrm{dBm}$ ) were used. An average WDM signal gain of $18.3 \mathrm{~dB}$ was measured in the spectral range of $1542-1560 \mathrm{~nm}$. The NF was found to increase sharply for wavelengths shorter than $1542 \mathrm{~nm}$ while it tends to decrease at longer wavelengths. The high NF at short wavelengths is mainly due to insufficient population inversion within the active medium and which is clearly evident from the sharp drop in signal gain. We observed large gain variation amongst the different elements, which is mainly due to the variation in core refractive index profiles and rare-earth doping concentrations of the nonidentical preform elements used. This could certainly be improved through further optimization of the preform fabrication process. The $\mathrm{LP}_{01}$ signal mode experienced higher gain than the $\mathrm{LP}_{11}$ mode and the differential modal gain (DMG) between the two guided modes varied by $1-6 \mathrm{~dB}$ over all 4 active fiber elements. Note that we used a simple step-index core design in the current fibres so this level of DMG is not unexpected, however careful tailoring of the doping profile within the individual elements (as illustrated in [17]) could certainly help in reducing the DMG in few-mode cladding-pumped MEFAs. Also there was no measurable inter-core crosstalk above the ASE levels observed between the neighboring fiber elements which is in agreement with our earlier observations $[9,10]$. It was not possible to measure the crosstalk below the ASE level due to the high loss in the unpumped cores at the signal wavelengths. As shown in Fig. 3, fiber element-3 (S3) shows the best performance in terms of differential modal gain (average $\mathrm{DMG} \sim 1.1 \mathrm{~dB}$, maximum DMG $\sim 3 \mathrm{~dB}$ ) and spectral gain flatness across the C-band and as such the detailed gain profiles for various pump power and input signal power levels were investigated for this element.

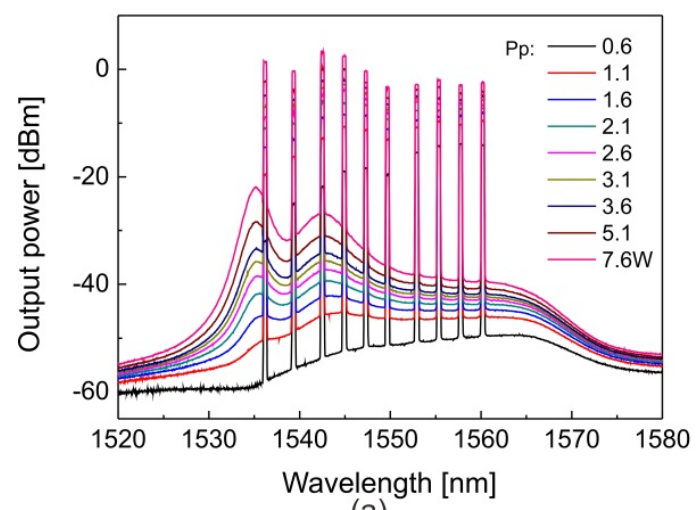

(a)

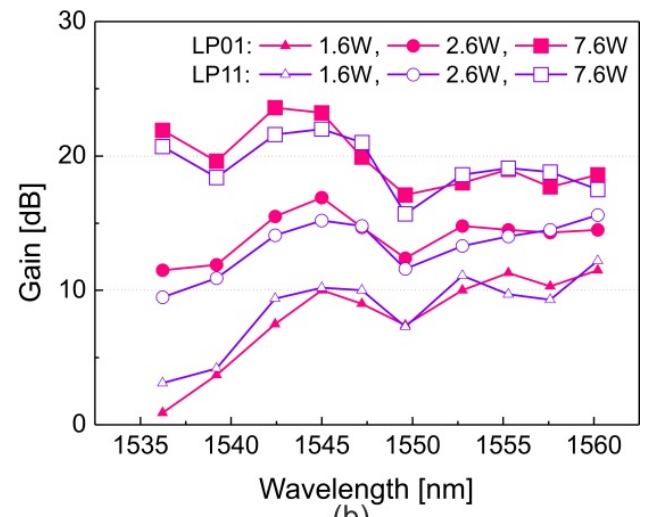

(b)

Fig. 4. (a) Output spectra of the $\mathrm{LP}_{01}$ mode after the mode demultiplexer and (b) modedependent gain for different pump powers with an input signal power of $-2.5 \mathrm{dBm} /$ mode (or $-12.5 \mathrm{dBm} / \mathrm{ch})$.

Figure 4(a) and 4(b) shows the output spectra and the mode dependent gain as a function of pump power at a fixed input signal power of $-2.5 \mathrm{dBm} /$ mode. As shown in Fig. 4(a), for low pump power $(\sim 0.6 \mathrm{~W})$ the amplified spontaneous emission (ASE) peak is located at $\sim 1565 \mathrm{~nm}$ and it shifts towards the shorter wavelength side with increasing pump power. A similar trend can be observed in the measured modal gain of the amplifier shown in Fig. 4(b). At low pump power the gain at the short wavelength edge was measured to be very low due to an insufficient inversion level. As the pump power was increased from $0.6 \mathrm{~W}$ to $7.6 \mathrm{~W}$, the gain of each of the guided modes increased due to the increased level of population inversion. It should be noted that the gain increment at the shorter wavelengths is higher compared to longer wavelengths resulting in a shift of gain peak from $1560 \mathrm{~nm}$ to $1545 \mathrm{~nm}$. 


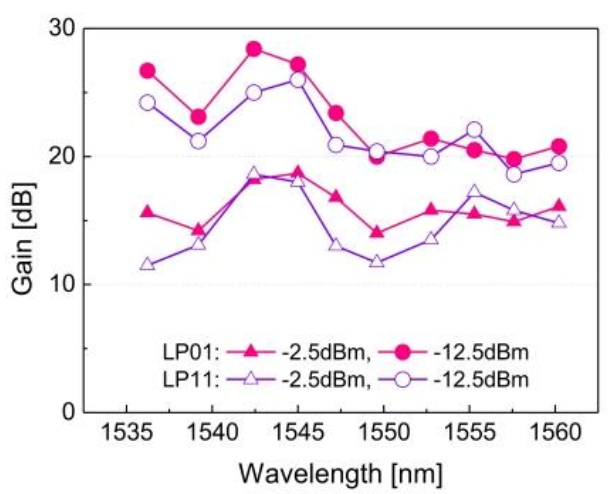

(a)

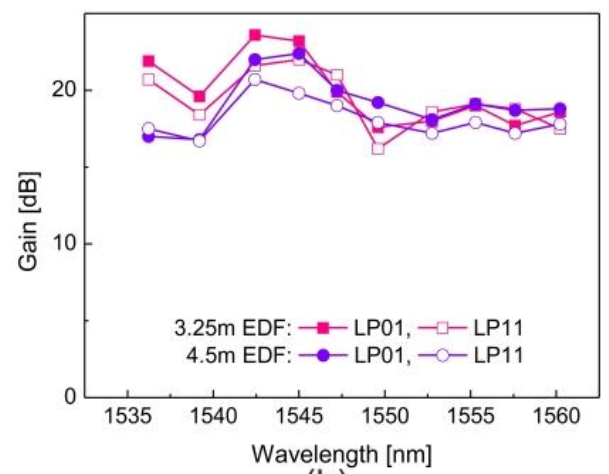

(b)

Fig. 5. (a) Mode-dependent gain for different input signal powers per mode at a fixed pump power of $3.6 \mathrm{~W}$ for $3.25 \mathrm{~m}$ of fiber, and (b) gain comparison between two different fiber lengths $(3.25 \mathrm{~m}$ and $4.5 \mathrm{~m})$ at a fixed pump power of $5.1 \mathrm{~W}$.

Figure 5(a) shows the amplifier gain variation for $-2.5 \mathrm{dBm}$ and $-12.5 \mathrm{dBm}$ input signal powers per mode at a fixed pump power of $3.6 \mathrm{~W}$. Both spatial modes experienced gain reduction with an increase in input signal power due to amplifier gain saturation. The DMG did not show much dependence on the input signal power and the maximum DMG remains at $\sim 3 \mathrm{~dB}$ for all the input signal powers investigated. We also investigated the length dependence of our 3M-MEFA. Figure 5(b) compares the gain spectra for two different fiber lengths $(3.25 \mathrm{~m}$ and $4.5 \mathrm{~m})$, for similar input signal powers $(-2.5 \mathrm{dBm}$ and $-3.3 \mathrm{dBm}$ per mode for $3.25 \mathrm{~m}$ and $4.5 \mathrm{~m}$ respectively) but at a fixed pump power of $5.1 \mathrm{~W}$. It can be seen that $3.25 \mathrm{~m}$ fiber exhibited about $5 \mathrm{~dB}$ higher gain in the shorter wavelength region as compared to that of the $4.5 \mathrm{~m}$ fiber indicating that the gain at shorter wavelength can be increased by optimizing the fiber length.

\section{Conclusions}

We have successfully demonstrated for the first time a few-mode multi-element fiber amplifier for space division multiplexed systems. An overall spatial multiplicity of 12 (3 spatial modes $\times 4$ signal fiber-elements) was obtained. An average signal gain of $18.3 \mathrm{~dB}$ and differential modal gain of $\sim 1-6 \mathrm{~dB}$ were achieved in the wavelength range of $1542-1560 \mathrm{~nm}$. This architecture can be further scaled both in terms of the number of elements and number of modes per-element. Improvements in gain differential between elements and modes can also be envisaged by tailoring the rare-earth doping profiles of the optical fiber. Improvements in NF should also be achieved by optimizing the element diameter/length and increasing the pump brightness.

\section{Acknowledgments}

This work was supported by the EPSRC grant EP/I01196X: Transforming the Future Internet: The Photonics Hyperhighway. The authors acknowledge OFS Denmark for providing the few-mode optical fibers and Tyndall National Institute at UCC in Cork, Ireland for providing the phase plates. 